\title{
Maunz/Dürig, Grundgesetz
}

\author{
Inhaltsübersicht
}

\author{
A. Gründerjahre \\ I. Der Beginn des Beginns \\ II. Dürigs Wurf \\ III. Maunzens Großarbeit \\ IV. Hinzutritt von Herzog und Scholz \\ B. Die weitere Entfaltung \\ I. Sachanforderungen \\ II. Personelles \\ III. Speziell zur Neukommentierung des Art. 1 \\ C. Allgemeine Charakteristik des Großkommentars „Maunz/Dürig“ \\ I. Konzeptionelle Vielfalt \\ II. Grund-Disziplin \\ III. Chance zu besonders einflußreichem Gestalten - Mühsal und Lust
}

\section{A. Gründerjahre}

\section{Der Beginn des Beginns}

Ein Gesamtwerk wie der vielbändige Kommentar zum Grundgesetz „Maunz/ Dürig“ hat seine eigene Geschichte. Der Kommentar genoß die Gnade der frühen Geburt: Das Grundgesetz war noch jung, das Bundesverfassungsgericht, wenig bekannt, machte seine ersten Schritte (die Karlsruher Taxis fuhren gewöhnlich statt zu ihm, zum Bundesgerichtshof). Daher bot sich die Chance einflussreichen Gestaltens. Wie Büchting ${ }^{1}$ - ein dem Kommentar bis zu seinem Ausscheiden lange Jahre engstens verbundener juristischer Lektor - berichtet, war der Verlagsvertrag 1952 zwar zunächst allein mit Theodor Maunz geschlossen worden; doch kam das Projekt erst in Schwung, als Günter Dürig Koautor geworden war. Allerdings musste man sich zunächst über die äußere Form einig werden. Trotz aller Bedenken gegen Loseblatt-Ausgaben schrieb Dürig ${ }^{2}$ dem Verlag, nur mit ,jeweils kleine(n) Abhandlungen, die selbständig in Einzellieferungen gebracht werden“, könne man vorankommen: „Ich habe es offengestanden langsam satt, dass nicht ich die Rechtsprechung beeinflusse“, sondern ,mich meinerseits mit Entscheidungen der Gerichte immer wieder im Manuskript neu auseinandersetzen muß, obwohl die Sache bei mir schon viel besser behandelt ist". In der Konsequenz fiel die Entscheidung für ein Loseblatt-Werk. Als später ein Kritiker auf die Nachteile

${ }^{1}$ Büchting, in: Juristen im Porträt, FS zum 225jährigen Jubiläum des Verlages C. H. Beck, 1988, S. 280 (283ff.).

${ }^{2}$ Vgl. Büchting (o. Fn. 1), S. 284. 
dieser Entscheidungsform hinwies, ließ Dürig drucken: „,Wo er recht hat, hat er recht".

\section{Dürigs Wurf}

Die erste Grundlieferung (1958) enthielt die Dürig'schen Kommentierungen von Art. 1 (Menschenwürde), Art. 2 (Freiheit) sowie Art. 104 (Richtervorbehalt). Im Verein hauptsächlich mit der (zum Leidwesen nicht nur des Verlags) erst sehr viel später - 1973 - erschienenen umfangreichen Kommentierung des Art. 3 (Gleichheit) wie auch weiterer grundgesetzlicher Normen prägte Dürig auf diese Weise einen entscheidenden Teil zumindest der ursprünglichen Gestalt des Kommentars. Er wurde darüber hinaus zum Baumeister eines maßgeblichen Verfassungsverständnisses überhaupt, so sehr sich dieses seither auch fortentwickelte oder sich mit prinzipiell anderen Konzeptionen auf dem wissenschaftlichen Turnierplatz traf. Seine monographischen Beiträge wurden berühmt. Begierig griff die deutsche juristische Welt nach dieser Architektur ${ }^{3}$ eines aus der Menschenwürde abgeleiteten umfassenden ,Wertesystems“; sei es oft voller Zustimmung, sei es eher skeptisch oder prinzipiell ablehnend, aber stets mit höchstem Respekt. Wie wichtig dies war - um nur ein Beispiel zu nennen -, hat rückblickend Böckenförde $e^{4}$ an Hand der Entstehung des Lüth-Urteils ${ }^{5}$ veranschaulicht. Begrüßenswert war denn auch die verlegerische Entscheidung, die Dürig'schen Kommentierungen des Art. 1 und des Art. 2 GG in einem Sonderdruck auf Dauer zu bewahren. ${ }^{6}$

\section{Maunzens Großarbeit}

Nach jenem eigentlichen Beginn des Werkes trat Theodor Maunz auf die Bühne; und dies mit einer gewaltigen Fülle von Einzelkommentierungen. Wie der Verfasser selbst beobachten konnte, betrieb Maunz sogar in seiner langen Zeit als Kultusminister oft schon sehr früh am Morgen seine Studien zum Kommentar, wonach er - wissenschaftlich gebadet, geölt und erfrischt - dem politischen Tageswerk mit gestärkter Gelassenheit ins Auge zu blicken vermochte. Und umgekehrt kam sein Reichtum an praktischer Erfahrung, gewürzt nicht nur mit dem Sinn für das Bleibende, auch mit einem Schuß Pragmatismus, seinen Kommentierungen zu gute - mitunter auch dadurch, dass sich bei Rückgriff auf deren Zusammenspiel eine gewisse Variationsbreite für die Lösung des jeweiligen Einzelfalls öffnet. Der

\footnotetext{
${ }^{3}$ Näher Lerche, in ders., Ronellenfitsch, Schmitt Glaeser, Graf Vitzthum, Zum Gedenken an Professor Dr. iur. Günter Dürig, 1999, S. 13 (13ff.).

${ }^{4}$ Böckenförde, Zur Lage der Grundrechtsdogmatik nach 40 Jahren Grundgesetz, 1990, S. 25 ff.; näher in der (ungedruckten) Diskussion nach diesem Vortrag.

${ }^{5}$ BVerfGE 7, 198. Zeitlich konnte sich das Votum zu dieser Entscheidung noch nicht auf Dürigs Kommentierung des Art. $1 \mathrm{im}$ „Maunz/Dürig“ selbst stützen, wohl aber auf den Dürigs Kommentierung im entscheidenden Teil vorwegnehmenden Beitrag „Der Grundrechtssatz von der Menschenwürde“ im AöR 81 (1956) S. 117 (117ff.).

${ }^{6}$ Verständlicherweise wurden diese Kommentierungen in den von Schmitt Glaeser und Häberle 1984 herausgegebenen Gesammelten Schriften Dürigs nicht aufgenommen, weil seinerzeit ohne weiteres im Kommentar zugänglich; aber für den Fall der Zweitbearbeitung wurde schon dort (vgl. S. 6) eine gesonderte Herausgabe der Dürig'schen Kommentierungen empfohlen.
} 
klaren Sprache und Systematik seiner oft wegweisenden Kommentierungen tat dies keinen Abbruch. Sein Sturz als Minister - die Gründe sind bekannt - hatte mit den Kommentierungen nichts zu tun. Wohl aber fand Maunz wiederum mehr Zeit für den Kommentar.

\section{Hinzutritt von Herzog und Scholz}

Den Begriff „Gründerjahre“ als Titel dieses Abschnitts darf, ja muß man großzügig verstehen, weil dem Kommentar ganz offenbar ein zumindest ebenso langes Leben beschieden sein wird wie dem Grundgesetz selbst, vom Verlag ganz zu schweigen. Daher breitet sich der Mantel der Gründerjahre auch noch aus auf den 1967 erfolgten Zutritt Roman Herzogs und die 1976 realisierte Erweiterung durch Rupert Scholz. Glücksfälle! Es war bewußt geworden, daß die monographische Anlage der Einzelbeiträge des Werkes durch Maunz und Dürig nicht allein bewältigt werden konnte, zumal angesichts der wegen einer Kriegsverletzung angeschlagenen Gesundheit Dürigs. Die Mitarbeit der Genannten realisierte sich in vielen, teilweise sehr umfangreichen Beiträgen, die den Ruhm des Werkes festigten. Darauf darf zurückgekommen werden.

\section{B. Die weitere Entfaltung}

\section{Sachanforderungen}

Dem Impetus des Kommentars konnte auch im weiteren Verlauf nur entsprochen werden, wenn er auf zusätzliche Schultern gelegt wurde. Die Notwendigkeit hier$\mathrm{zu}$ verstärkte sich durch verfassungsrelevante Entwicklungen verschiedenster Art, die untereinander verknüpft sind:

Am Handgreiflichsten zeigt sich dies wohl angesichts der vorgenommenen zahlreichen formellen (= geschriebenen) Änderungen des Grundgesetzes. Diese Änderungen erwachsen teilweise aus ganz prinzipiellen Fortentwicklungen, von den frühen großen Reformen wie Wehrverfassung, Notstandsverfassung, erste große Finanzreform, bis zuletzt zur umfassenden Föderalismusreform (deren Kommentierung im Wesentlichen erst bevorsteht). Besondere Aufmerksamkeit verlangten die verschiedenen verfassungsrechtlichen Stufen, auf denen der Gang zur Europäischen Union vollzogen wurde, und deren Auswirkungen. Hier galt und gilt es auch, an den Kern der Staatsverfassung rührende Fragen zu beantworten. Für den Vollzug der Wiedervereinigung, die ihrerseits das Grundgesetz gerade nicht als Ganzes von sich warf, sondern sich seiner bediente und es inhaltlich prinzipiell akzeptierte, waren schon ursprüngliche Erläuterungen im „Maunz/Dürig“ wichtig; doch stellten sich aus diesem Anlaß zugleich neue Kommentierungsaufgaben. Und ferner: Manche formelle Änderungen speziell im Grundrechtsteil mussten und müssen berücksichtigt werden; auch dort, wo der Eindruck vorherrscht, gewisse Detailänderungen besäßen im Grunde nicht inhaltliche Verfassungshöhe, sondern verdankten ihren formellen Verfassungsrang spezifischen politischen Absicherungserwägungen. 
Bei der Erläuterung formeller Verfassungsnormen spiegelt sich zugleich die Fülle allgemeinerer Entwicklungen und Fragestellungen; etwa in sozialer, wirtschaftlich-technischer, medizinischer und sonstiger wissenschaftsgebundener, sicherungsrechtlicher, datenschutzrechtlicher, staatkirchenrechtlicher Art sowie z.B. solche aus dem organisatorisch politisch-parlamentarischen Feld. Nur teilweise, wie etwa bei gewissen sogen. Privatisierungen, schlägt sich dieser Ansturm in formellen Verfassungsänderungen nieder; er kann aber auch sonst nicht negiert werden - was nicht heiß, Trends schlicht zu akzeptieren, wohl aber, neu überprüfte Antworten zu geben.

Von früh an - erinnert sei an das schon genannte Lüth-Urteil ${ }^{7}$ - konnte der Kommentar zudem nicht vorbeigehen an der Einflussnahme des Verfassungsrechts auf das unterverfassungsrechtliche Recht. Sie hat oft geradezu beherrschenden Charakter, läßt sich aber auf keine einfache Formel bringen. Umgekehrt muß die Relevanz des unterverfassungsrechtlichen Rechts für die Prägung wie auch mitunter stille inhaltliche Fortentwicklung des Verfassungsrechts beachtet werden. Dadurch entstehen schwierige Balanceaufgaben. Ausbreitung und Veränderung des sekundären Europarechts, u. U. auch des internationalen Rechts kommen hinzu, weil und soweit sie grundgesetzliche Fragen auslösen.

Vor allem aber sind es wohl zwei durchgängige Gegebenheiten, die den Kommentatoren einiges abfordern. Einmal müssen sie, trotz der Panzerung des zu Kommentierenden in geschriebenen Formulierungen, die dahinter wirkende vielfache Existenz ungeschriebenen Verfassungsrechts beachten, und das heißt dieses zunächst ans Licht bringen. Das ist keine schlicht interpretierende Aufgabe. Dennoch darf sich der Titel des „Maunz/Dürig“ nicht vom „Kommentar“ unter der Hand verändern etwa in „Bermerkungen zum Verfassungsrecht“.

Und zum andern wird in den vielleicht sogar meisten Feldern deutlich, dass es Norm-Kollisionen sind, aus denen die entscheidenden Probleme erwachsen. Ihnen muß sich jeder Kommentar stellen; glatte Ober-Formeln, wie etwa „Einheit der Verfassung" helfen da wenig, so beliebt sie anderswo sind. Historische und rechtsvergleichende Erfahrungsschätze sind wohl eine größere Hilfe. ${ }^{8}$

Allen diesen Sachanforderungen in idealer Weise nachzukommen bedürfte eines Ideal-Kommentators. Den kann es nicht geben. Der Verleger schläft bekanntlich nie wirklich, also kann er sich einen solchen auch nicht erträumen. An Wachträumen leidet er als Sportler ohnehin nicht.

Aber - und dies dürfte anerkannt sein - dem Range und der Standfestigkeit des Kommentars in Theorie und Praxis konnte dadurch genügt werden, dass als Mitautoren Gelehrte aufs Schiff geholt wurden, denen man - etwas flott ausgedrückt - verfassungsrechtlich nichts vormachen kann. Extreme Positionen finden hier keine Heimstatt, ebenso wenig verspielte Theorien.

Unter Einschluß der schon oben (I. 4.) genannten ersten Erweiterung des Autorenkreises darf gesagt werden:

\footnotetext{
${ }^{7}$ Fn. 5.

${ }^{8}$ Dem Kommentar ist - neben einem umfangreichen Sachverzeichnis - eine große Reihe verfassungsrelevanter Normenwerke beigegeben. Dieser Begleittext wird von Lieferung zu Lieferung aktualisiert bzw. ergänzt.
} 


\section{Personelles}

Roman Herzog, der ja schon lange vor seinen Ämtern u.a. als Präsident des Bundesverfassungsgerichts und dann als Bundespräsident zum Kommentar als Gelehrter gewonnen werden konnte, hat sich, wenn wir recht sehen, in seiner Amtszeit als Bundespräsident durchaus an seine eigene Kommentierung der Artikel über den Bundespräsidenten gehalten. Was blieb ihm auch übrig! Er hat zudem von Beginn an zahlreiche weitere Normen, etwa zu den Meinungs- und Medienfreiheiten, zur Bundesregierung, zu Rechtsprechungsbereichen, zum Verteidigungsfall u.a.m., erläutert.

Der gegenwärtige Präsident des Bundesverfassungsgerichts, Hans-Jürgen Papier, pflegt seine besondere Nähe zu den vielfältigen Fragen des Eigentumschutzes, sowie des Erbrechts, der Unverletzlichkeit der Wohnung, der Amtshaftung u.a.m., von den Grundlinien bis ins spannende und wichtige Detail. Bundesminister a.D. Rupert Scholz, der den Kommentar schon früh neben seiner gelehrten auch aus jenen Erfahrungen bereicherte, die er aus verschiedenen politischen Feldern zog, sind zentrale und reichhaltige Erläuterungen zu danken; so etwa, um nur Einiges herauszugreifen, jene zur Vereinigungs- und zur Berufsfreiheit (letztere mit jüngster umfassender Aktualisierung), zum Schutz der natürlichen Lebensgrundlagen, $\mathrm{zu}$ den Vorschriften über den Bundesrat und insbesondere auch zur Position des Grundgesetzes zur Europäischen Union. Hans H. Klein, früherer Bundesverfassungsrichter, schöpft ebenfalls zusätzlich aus eigenen politischen Erfahrungen und kommentierte schon bislang ausgedehnte Normenkomplexe, wie sie in den Grundgesetzvorschriften zu den politischen Parteien, zum Bundestag einschließlich etwa des Rechts der Untersuchungsausschüsse u.a. geformt sind, wie auch Art. 17 samt Art. 45c, (Petitionsrecht), Art. 18 (Verwirkung). Der Richter des Bundesverfassungsgerichts Udo Di Fabio stellte sich neu den Fluten des Art. 2 und rettet Ertrinkende.

Staatsrechtslehrer mit reicher Erfahrung in der - insbesondere auch prozessualen - Praxis traten hinzu. So schuldet man die wegweisenden Kommentierungen von Art. 6 (Ehe und Familie) sowie von Art. 7 Abs. 1 bis 4, Art. 141 (Schulwesen, Religionsunterricht) Peter Badura, mit der dieser führende Gelehrte seine Arbeit am „Maunz/Dürig“ begann. Albrecht Randelzhofers internationalrechtliche Interessen finden ihre Resonanz in den Erläuterungen des Art. 16a (Asylrecht) sowie des Art. 24 (internationale Einrichtungen). Höchst wirkungsvoll erwiesen sich auch die aus neuen Perspektiven entfalteten Kommentierungen der Rechtschutz-Artikel 19 Abs. 4 sowie 103 durch Eberhard Schmidt-Aßmann; hier war zugleich die spezifische Verflechtung mit einfachem Gesetzesrecht zu bewältigen. Stefan Korioth hat sich bisher den Tiefen der staatskirchenrechtlichen Aussagen sowie dem Art. 30 (prinzipielle Kompetenzverteilung zwischen Bund und Ländern) zugewandt. Auf Matthias Herdegens Neukommentierung des Art. 1 wird sogleich zu sprechen zu kommen sein. Von ihm stammt auch die neue Erläuterung des Art. 26 (Verbot des Angriffskriegs). Der Verfasser bemühte sich, Basisvorstellungen und Einzelkomplexe zur verwaltungsmäßigen Ausführung der Bundesgesetze (Art. 83 ff.) - wiewohl nicht vollständig - zu klären. Künftig 
wird die Föderalismusreform gerade auch im Verwaltungsbereich zu beachten sein, die überhaupt zu umfangreichen Neubearbeitungen führen wird müssen. In junger Zeit legten erstmalig Bernd Greszick (Bundesstaatlichkeit im Sinn von Art. 20) und Arnd Uhle (Fortgelten früheren Bundesrechts, Art. 125a) Kommentierungen vor.

\section{Speziell zur Neukommentierung des Art. 1}

Wie berichtet hat der großartige Beginn des Ganzen durch die Kommentierung des Art. 1 durch Dürig Praxis und Theorie aufs Intensivste angeregt, ja bestimmt: Dies zumal, als sich die Auswirkungen auf breite Bereiche des Verfassungsrechts erstrecken, wie dies bei Entwurf eines „Wertsystems“ nun einmal beansprucht wird. Daher ist es verständlich, dass die Neukommentierung des Art. 1 durch Herdegen (inzwischen ihrerseits aktualisiert) die wohl breiteste Diskussion aller Neukommentierungen auslöste. Aus diesem Grund sei sie als Beispiel für die Lebendigkeit des Kommentars kurz herausgegriffen, wie immer man inhaltlich zu ihr stehen mag.

So sehr Herdegen Dürig'sche Ansätze und Deutungen aufnimmt, so sehr sind doch Unterschiede erkennbar. Er wendet sich gegen ein geschlossenes grundrechtliches Wert- und Anspruchssystem; eine absolute Dominanz der Garantie der Menschenwürde insbesondere gegenüber dem Lebensschutz wird verneint; die „Konkretisierung“ der Menschenwürde - darauf liegt der Akzent - sei der Ermittlung des Gewährleistungsgehalts (Unantastbarkeit) vorgeordnet. Wie etwa beim pränatalen Würdeschutz deutlich werde, sei eine ,prozeßhafte“ Betrachtung des Würdeschutzes - mit entwicklungsabhängiger Intensität eines bestehenden Achtungs- und Schutzanspruchs - angebracht. Absolute Geltung habe erst der so konkretisierte Würdeanspruch.

Die an diese Ausgangssicht und ihre konkreten Folgerungen anschließende Auseinandersetzung, auch mit entschieden kritischen Stimmen (etwa Böckenförde), ist ein wichtiger Bestandteil der gegenwärtigen allgemeineren Verfassungsdiskussion. Auch viele andere Neukommentierungen bewegen sich nicht nur auf schon gelegten Gleisen. Doch kann dies hier nicht ausgemalt werden. Der Verlag hat sich hierbei, sieht man richtig, stets zurückgehalten; aber an der fortdauernden Lebendigkeit wie Standfestigkeit des Kommentars muß er natürlich interessiert sein.

\section{Allgemeine Charakteristik des Großkommentars „Maunz/Dürig“}

\section{Konzeptionelle Vielfalt}

In der berichteten Geschichte des Kommentars ist begründet, daß das Gesamtwerk nicht wie bei anderen Großkommentaren nach einem einheitlichen Plan geformt wurde, zu dessen Realisierung sich von Anfang an eine größere oder große Reihe von Autoren zusammengefunden hätte. Schon zu Beginn wäre es abwegig gewesen, sich etwa Maunz vorzustellen, wie er auch nur ein winziges Peitschchen über Dürig hätte schwingen wollen. Auch die später eintretenden Autoren ver- 
wirklichten in Systematik und Methode ihren je eigenen Stil bei der Bearbeitung der von ihnen übernommenen Teile. Von einigen vorgegebenen Eingrenzungen und Anforderungen abgesehen, konnte daher eine Kompaktheit, eine Art PlanErfüllung der angedeuteten Art nicht entstehen.

Vielleicht hätte sich der Verleger eine größere Geschlossenheit gewünscht. Immerhin sind in jüngerer Zeit die erwähnten äußeren Anforderungen verstärkt worden. Aber wenn man das richtig fühlt, ist es doch auch für den Verleger und seine mit dem Kommentar speziell verbundenen hochgeschätzten Mitarbeiter am wichtigsten, daß der Kommentar angesichts des referierten Profils und Ranges der Autoren seinen ganz besonderen Platz auch künftig zu behaupten vermag. Formale Anforderungen sollen dies unterstützen. Einzuberechnen ist zudem, daß es just jene gewisse konzeptionelle Freiheit sein dürfte, die mehr als nur ein Zuckerbrot dazu beiträgt, die Mitarbeit am Kommentar attraktiv zu machen. Der Verleger hat freilich die - erst im Rückblick dankbare - Aufgabe, dafür zu sorgen, daß die Dinge im Äußeren nicht zu sehr auseinanderlaufen. Auch hierzu dienen die regelmäßigen Redaktionskonferenzen, bei denen eine angenehm offene Aussprache stattfindet; sie hatten in jüngerer Zeit indessen auch und vor allem den Sinn, die Gewinnung der neuen Mitautoren vorzubereiten und die gewaltige Gesamtarbeit aufzuteilen - effektiv und ohne Tücken. Die ebenfalls erst in jüngerer Zeit installierte Herausgeberschaft (Herzog-Scholz-Herdegen-Klein) ${ }^{9}$ bietet zu alledem eine große Hilfe.

\section{Grund-Disziplin}

Wie oft mag sich die Feder eines Autors dazu hingezogen fühlen, in die Kommentierung eigene Wunschvorstellungen einfließen $\mathrm{zu}$ lassen und sie als geltendes Verfassungsrecht auszugeben! Gilt doch gerade das Grundgesetz mit seinen oft vagen Formulierungen in vielerlei Augen als Wunschtüte. Und der Kommentar verfügt zwar über keine Interpretations"macht", aber sein Wort wird überall gehört. Das mag eine gewisse Verführung bedeuten. Gleichwohl: Wenn man recht sieht, widerstanden die Autoren des „Maunz/Dürig“ dieser Verführung wohl stets - was natürlich eine als solche deutlich gemachte rechtspolitische Kritik nicht ausschließt. Erst recht wird dadurch Kritik an vorhandenen gerichtlichen, auch verfassungsgerichtlichen Entscheidungslinien nicht ausgeschlossen: Selbst die generelle Bindungswirkung eines Teils der verfassungsgerichtlichen Entscheidungen hindert wissenschaftliche Kritik nicht. Allein, ohne jene Grund-Disziplin hätte sich der Kommentar nicht die außerordentliche Akzeptanz erobern können, die ihn auszeichnet. Man kann das Maß dieser Wirkkraft auch daran ablesen, wie häufig der Kommentar in den Entscheidungen des BVerfG seit jeher zitiert wird, wiewohl speziell verfassungsrechtliche Literatur in einem großen Teil dieser Entscheidungen eine nur sehr selektive Zitierung findet.

\footnotetext{
${ }^{9}$ Geschäfsführend sind dabei die Herren Herdegen und Klein.
} 


\section{Chance zu besonders einflussreichem Gestalten - Mühsal und Lust}

Die erwähnte besondere Akzeptanz des Kommentars erklärt sich aus einer ganzen Reihe von Umständen: Neben jener Grund-Disziplin etwa aus Profil und Rang der Autoren, Ansehen des Verlags, wegweisender Kraft der Erläuterungen, deren Genauigkeit, verbunden mit dem Ziel, mehr oder minder durchgehende Strukturen der einzelnen Teilmaterien aufzudecken. In diesem Sinn beherrscht theoriegestützte Praxiszugewandtheit das Ganze.

Die so erarbeitete Akzeptanz eröffnet, ähnlich wie dies schon die Anfänge des Kommentars charakterisierte, auch heute noch die Chance $\mathrm{zu}$ besonders einflussreichem wissenschaftlichen Gestalten. Das inspiriert wohl jeden Mitautor. Die Mühsal des Kommentierens verwandelt sich auf diese Weise zugleich zu einer Art Lust, ohne freilich in ihr aufzugehen. 